\section{HIV protease inhibitors and atherosclerosis}

\author{
David Y. Hui
}

Department of Pathology, University of Cincinnati College of Medicine, Cincinnati, Ohio, USA

J. Clin. Invest. 111:317-318 (2003). doi:10.1172/JCI200317746.

The advent of highly active antiretroviral therapy (HAART), including the use of HIV protease inhibitors (PIs) has significantly reduced the morbidity and mortality of AIDS in HIV infected patients. Unfortunately, the adverse effects of PIs, including dyslipidemia, lipodystrophy, insulin resistance, and premature atherosclerosis, are cause for concern for their use in chronic management of HIV infection $(1,2)$. At present, the relationship between HAART and premature atherosclerosis in HIV-infected patients is unclear. Lipid abnormalities and insulin resistance induced by PIs can certainly increase the risk of premature atherosclerosis. Chronic inflammation associated with HIV infection, particularly the increased level of $C$ reactive protein (3), may also contribute to accelerated atherosclerosis in these patients. Whether the treatment regimen alone directly contributes to accelerated atherosclerosis, however, has not been scrutinized vigorously.

In this issue of the JCI, Dressman et al. showed that HIV PIs directly promote atherosclerosis in mice (4). Importantly, the authors demonstrated that PIs at a dosage that did not induce hyperlipidemia are potent promoters of atherosclerosis, thus providing the first evidence for a direct effect of PIs in atherosclerosis. Although the in vivo studies were performed in genetically-engineered mouse models

Address correspondence to: David Y. Hui, Department of Pathology, University of Cincinnati College of Medicine, 231 Albert Sabin Way, Cincinnati, Ohio 45267-0529, USA.

Phone: (513) 558-9152; Fax: (513) 558-2141; E-mail: huidy@email.uc.edu.

Conflict of interest: The author has declared that no conflict of interest exists.

Nonstandard abbreviations used: highly active antiretroviral therapy (HAART); protease inhibitor $(\mathrm{PI})$. of atherosclerosis, their in vitro results that PIs also induced foam cell formation in a human macrophage cell line are of significance.

The study by Dressman et al. (4) also suggested that PIs directly promote atherosclerosis by inducing CD36 gene expression in macrophages. This observation is consistent with the documented role of macrophage CD36 in atherosclerosis (5). However, the results were in direct contrast to another study, which reported that PIs decreased monocyte CD36 levels in healthy volunteers and HIV-infected individuals (6). The discrepancy between the two studies may be due to differences in experimental design. Whereas Dressman et al. examined CD36 mRNA and protein expression in mouse macrophages and differentiated human THP-1 cells, Serghides and colleagues focused on $\mathrm{CD} 36$
Figure 1 mature form of SREBP. expression in human monocytes and undifferentiated THP- 1 cells. Thus, it is possible that PIs have opposite effects on CD36 expression in monocytes and macrophages. If this turns out to be the case, PI-induced down regulation of CD36 expression in monocytes and other cell types may be responsible for impaired glucose tolerance, insulin resistance, and hyperlipidemia (6), whereas their up-regulation of macrophage $\mathrm{CD} 36$ may promote foam cell formation and atherosclerosis (4). CD36 is a major fatty acid transporter in tissues with high metabolic capacity (5). Its down-regulation in tissues such as the heart, adipose, and skeletal muscle would impair fatty acid utilization and decrease insulin responsiveness in these tissues, thus resulting in glucose intolerance, insulin resistance, and hyperlipidemia. These two effects may act synergistically in promoting premature atherosclerosis.

Dressman et al. (4) also found that PI-induced macrophage CD36 expression is related to the activation of PPAR $\gamma$ in a protein kinase $C$ dependent manner. How compounds designed to inhibit protease activity

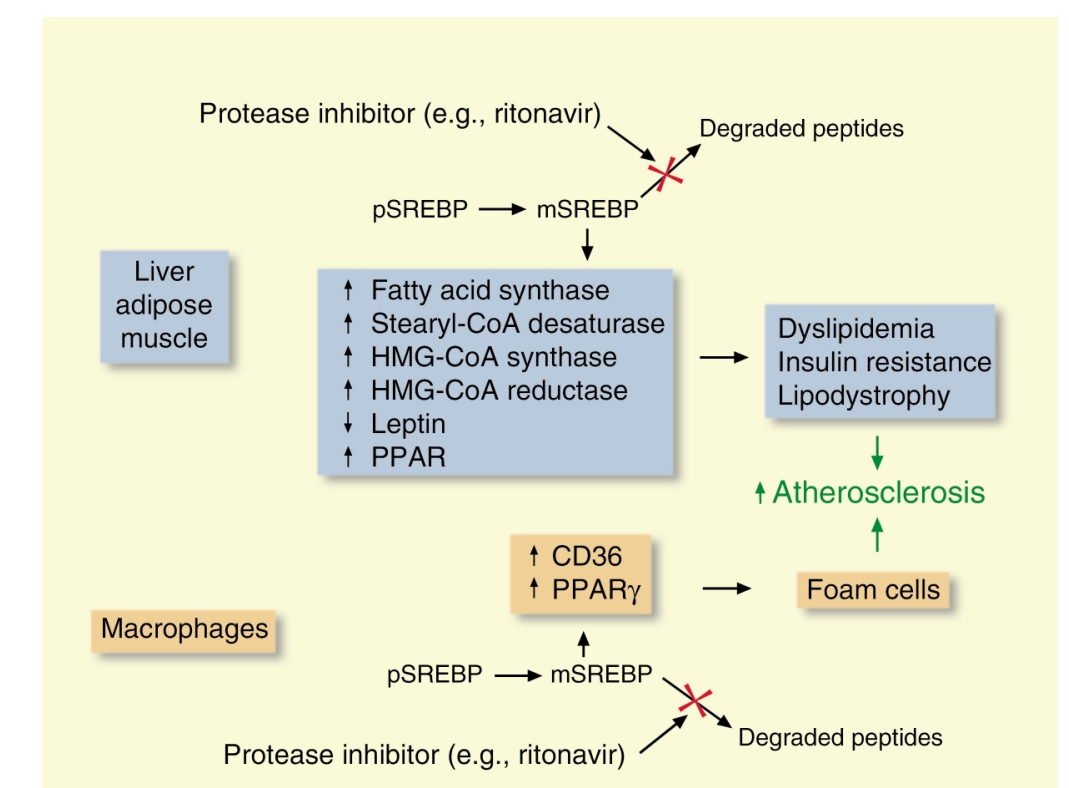

Schematic diagram depicting mechanisms by which HIV protease inhibitors may promote atherosclerosis through increased accumulation of SREBP. pSREBP, precursor form of SREBP; mSREBP, 
influence protein kinase $\mathrm{C}$ activity and activate PPAR $\gamma$ gene transcription remains unclear. However, it is now established that PIs, particularly ritonavir, are also inhibitors of proteasomemediated protein degradation pathways $(7,8)$. These include their inhibition of activated SREBP degradation, resulting in constitutive activation of SREBP-1 and SREBP-2 responsive genes (8). Since activated SREBPs are also promoters of PPAR $\gamma$ expression (9), the PI-induced PPAR $\gamma$ and CD36 expression may be mediated via PI-induced activated SREBP accumulation in the macrophage nucleus. This hypothesis would predict that PIs promote atherosclerosis through two mechanisms, both involving increased SREBP activity (Figure 1). One mechanism is through PI-induced metabolic complications that increased the risk of atherosclerosis; the other mechanism is a direct effect of PIs on macrophage foam cell formation. If this hypothesis is proven correct, then novel compounds that inactivate SREBP activity may be designed and used to alleviate both metabolic and cellular complications that promote cardiovascular events associated with HAART.

1. Carr, A., Samaras, K., Chisholm, D.J., and Cooper, D.A. 1998. Pathogenesis of HIV-1 protease inhibitor-associated peripheral lipodystrophy, hyperlipidaemia, and insulin resistance. Lancet 351:1881-1883.

2. Henry, K., et al. 1998. Severe premature coronary artery disease with protease inhibitors. Lancet. 351:1328.

3. Jahoor, F., et al. 1999. The acute-phase protein response to human immunodeficiency virus infection in human subjects. Am. J. Physiol. 276:E1092-E1098.

4. Dressman, J., et al. 2003. HIV protease inhibitors promote atherosclerotic lesion formation independent of dyslipidemia by increasing CD36- dependent cholesteryl ester accumulation in macrophages. J. Clin. Invest. 111:389-397. doi:10.1172/200316261

5. Febbraio, M., Hajjar, D.P., and Silverstein, R.L. 2001. CD36: a class scavenger receptor involved in angiogenesis, atherosclerosis, inflammation, and lipid metabolism. J. Clin. Invest. 108:785-791. doi:10.1172/JCI200114006.

6. Serghides, L., Nathoo, S., Walmsley, S., and Kain K.C. 2002. CD36 deficiency induced by antiretroviral therapy. AIDS. 16:353-358.

7. Andre, P., et al. 1998. An inhibitor of HIV-1 protease modulates proteasome activity, antigen presentation, and $\mathrm{T}$ cell responses. Proc. Natl. Acad. Sci. USA. 95:13120-13124.

8. Riddle, T.M, Kuhel, D.G., Woollett, L.A., Fichtenbaum, C. J., and Hui, D.Y. 2001. HIV protease inhibitor induces fatty acid and sterol biosynthesis in liver and adipose tissues due to the accumulation of activated sterol regulatory element binding proteins in the nucleus. J. Biol. Chem 276:37514-37519.

9. Fajas, L., et al. 1999. Regulation of peroxisome proliferator-activated receptor gamma expression by adipocyte differentiation and determination factor 1 /sterol regulatory element binding protein 1: implications for adipocyte differentiation and metabolism. Mol. Cell. Biol. 19:5495-5503. 